\title{
FRATURA TORACOLOMBAR DO TIPO EXPLOSÃO: CORRELAÇÃO ENTRE CIFOSE E FUNÇÃO APÓS TRATAMENTO CONSERVADOR
}

\author{
THORACOLUMBAR BURST FRACTURES: CORRELATION BETWEEN \\ KYPHOSIS AND FUNCTION POST NON-OPERATIVE TREATMENT
}

Osmar Avanzi', Robert Meves², Maria Fernanda Silber Caffaro ${ }^{3}$, João Paris Buarque de Hollanda ${ }^{4}$, Marcelo Queiroz ${ }^{4}$

\section{RESUMO}

Objetivo: Avaliar a correlação entre cifose pós-traumática e função nos pacientes submetidos ao tratamento conservador das fraturas toracolombares do tipo explosão. Métodos: Realizado estudo retrospectivo segundo os critérios de Denis e Magerl classificados como subtipo A3 em 36 pacientes que preencheram o critério de inclusão para esta amostra e submetidos à aplicação de gesso antigravitacional ou órtese toracolombossacra (TLSO). A média de idade dos pacientes estudados foi de 50,83 anos, com mínimo de 13 e máximo de 83 anos, sendo 20 do sexo masculino e 16 do feminino. O resultado do tratamento foi avaliado com base no questionário de qualidade de vida Short Form-36 (SF-36) e nas escalas de dor e trabalho de Denis e o quadro clínico neurológico conforme a classificação de Frankel. A quantificação da dor foi realizada com base na escala visual analógica da dor (EVA) A mensuração da cifose residual foi obtida pelo método de Cobb à admissão e ao final do seguimento. Mostrar o grau maior, menor e a média na admissão e no final do tratamento. Resultados: Foi observada fraca correlação positiva $(r=0,563 ; p<0,001)$ entre a cifose residual e o escore de dor (EVA). Não houve correlação entre cifose final e o SF-36 e as escalas de Denis ( $p>0,05)$. Conclusão: Não existe evidente relação entre a cifose residual e o desfecho funcional e de sintomas dos pacientes.

Descritores - Fraturas da coluna vertebral/ terapia; Fraturas da coluna vertebral/ complicações; Cifose; Resultado de tratamento

\section{ABSTRACT}

Objective: To assess the correlation between kyphosis and post-traumatic symptoms in patients undergoing conservative treatment for thoracolumbar burst fractures. Methods: A retrospective study was carried out with 36 patients meeting the inclusion criteria for this kind of fracture classified as Denis and Magerl's subtype A3 and treated with anti-gravitational casting or TLSO. The mean age of patients was 50.83 years, ranging from 13 to 83 years, being 20 male and 16 female subjects. The treatment outcome was evaluated based on the SF-36 questionnaire, on Denis scores for pain and work and Frankel clinical and neurological scale. The quantification of pain was based on the visual analogue scale for pain. The measurement of the residual kyphosis was obtained by the Cobb method at admission and at the end of the follow-up. Results: A weak positive correlation ( $r=0.563$; $p<0.001$ ) was found between residual kyphosis and pain score (EVA). No correlation was found between final kyphosis and SF-36 and Denis scores $(p>0.05)$. Conclusion: There is no evident correlation between residual kyphosis, functional outcome and patients' symptoms.

Keywords - Spinal fractures/therapy; Spinal fractures/complications; Kyphosis; Treatment outcome

1 - Diretor do Departamento de Ortopedia e Traumatologia, Faculdade de Ciências Médicas, Santa Casa de Misericórdia de São Paulo (SCMSP), São Paulo, Brasil.

2 - Chefe do Grupo de Coluna do Departamento de Ortopedia e Traumatologia, Faculdade de Ciências Médicas, Santa Casa de Misericórdia de São Paulo (SCMSP), São Paulo, Brasil.

3 - Assistente do Grupo de Coluna do Departamento de Ortopedia e Traumatologia, Faculdade de Ciências Médicas, Santa Casa de Misericórdia de São Paulo (SCMSP), São Paulo, Brasil.

4 - Médico Residente do Departamento de Ortopedia e Traumatologia, Faculdade de Ciências Médicas, Santa Casa de Misericórdia de São Paulo (SCMSP), São Paulo, Brasil.

Trabalho realizado pelo Grupo de Coluna Vertebral do Pavilhão "Fernandinho Simonsen” do Departamento de Ortopedia e Traumatologia, Faculdade de Ciências Médicas, Santa Casa de Misericórdia de São Paulo (SCMSP).

Correspondência: Robert Meves, Rua Dr. Cesário Mota Jr., 112 - 01277-900 - São Paulo (SP) - Brasil. E-mail: robertmeves@hotmail.com 


\section{INTRODUÇÃO}

A fratura toracolombar do tipo explosão resulta de compressão axial com ruptura das colunas anterior e média do corpo vertebral, apresentando retropulsão de fragmento ósseo para o interior do canal vertebral e aumento da distância interpedicular ${ }^{(1)}$. Essa região é local comum de lesões, com incidência variando de $10 \%$ a $45 \%^{(1-3)}$.

Esse tipo de lesão é frequente em indivíduos jovens em idade produtiva, sendo resultado de trauma de alta energia. Ela pode estar associada a outras lesões, tais como fraturas em outros níveis da coluna vertebral, de membros, da bacia e lesões em tórax e abdômen ${ }^{(4)}$.

Cerca de $90 \%$ de todas as fraturas da coluna vertebral ocorrem entre T11 e L4 e em torno de 14 a 17\% são classificadas como tipo explosão. Essa região é sensível a lesão por três razões: a perda da estabilização proporcionada pelas costelas e musculatura torácica; a transição de curvatura torácica cifótica para lombar lordótica e a mudança na orientação das facetas articulares de coronal na coluna torácica para sagital na coluna lombar ${ }^{(4)}$.

Alguns pacientes com fratura do tipo explosão desenvolvem instabilidade mecânica progressiva, caracterizada por aumento da cifose, dor nas costas e sequela neurológica $^{(1,5-7)}$. Watson-Jones ${ }^{(5)}$ considerava o tratamento com simples repouso no leito por algumas semanas como inadequado, pois a deformidade em cifose era progressiva, levando à instabilidade mecânica da coluna, resultando em sintomas como dor local persistente e alterações degenerativas precoces. Concluiu que a solução seria tratar a lesão como qualquer fratura ou luxação de outra região do corpo, por redução em extensão e imobilização com colete gessado até que houvesse consolidação.

$\mathrm{Nicoll}^{(6)}$ referiu que a recorrência da deformidade após redução e imobilização prolongada era mais comum que o esperado e definiu como fatores mais importantes para a perda da redução a cominuição do corpo vertebral, lesão do disco intervertebral e ruptura do ligamento interespinhoso. Afirmava ainda que bom resultado anatômico era indispensável para bom resultado funcional. Tratava as fraturas estáveis (encunhamento anterior e lateral, fraturas de lâmina acima de L4) de maneira "funcional", com repouso no leito e exercícios progressivos, e fraturas instáveis (fraturaluxação e fraturas de lâmina abaixo de L4) com órteses em posição neutra ou fisiológica do tronco.
As fraturas instáveis com cifose $>30^{\circ}$, perda de altura $>50 \%$, compressão do canal vertebral $>50 \%$, lesão do complexo ligamentar posterior e associadas a déficit neurológico em geral apresentam indicação de tratamento cirúrgico pela maioria dos autores ${ }^{(8-17)}$.

Entretanto, nas fraturas toracolombares do tipo explosão sem sinais de instabilidade e com exame neurológico normal. o tratamento é controverso. Os que defendem o procedimento cirúrgico referem que este proporciona melhor correção da cifose, diminuindo a dor e futuras alterações degenerativas, previne deterioração neurológica e recorrência da deformidade, reduz os períodos de imobilização, repouso e internação ${ }^{(11,13,14)}$. Em contrapartida, outros autores relatam como vantagens do tratamento conservador bom resultado funcional no longo seguimento, pequena progressão da deformidade, baixa incidência de déficit neurológico, menor custo e menores complicações quando comparado com o tratamento cirúrgico ${ }^{(2-23)}$.

A despeito do grande número de publicações sobre o tratamento conservador da fratura toracolombar tipo explosão, a maioria dos trabalhos não aborda ou discute a relação entre a cifose final e o desfecho funcional nos pacientes tratados de modo conservador. Quando observamos essa análise, em geral, os autores utilizam questionários próprios ou a interpretação subjetiva da dor pelo paciente, dificultando a interpretação dos resultados ${ }^{(14,16,23)}$.

O objetivo deste estudo foi observar a correlação entre a cifose pós-traumática e a função e a sintomatologia nos pacientes submetidos ao tratamento conservador das fraturas toracolombares do tipo explosão.

\section{MÉTODOS}

Foi realizado estudo retrospectivo, de corte transversal, no Departamento de Ortopedia e Traumatologia da Santa Casa de Misericórdia de São Paulo. Foram coletados todos os prontuários, radiografias e tomografias computadorizadas (TC) nos cortes axiais dos portadores de fratura tipo explosão da coluna toracolombar, segundo os critérios de Denis ${ }^{(1)}$, no Serviço de Arquivo Médico e Estatística (SAME), internados entre 1991 e 2008. Este projeto de pesquisa foi aprovado pelo Comitê de Ética em Pesquisa do Serviço de Ortopedia e Traumatologia de Santa Casa de São Paulo.

Foram incluídos neste trabalho os pacientes com fratura tipo explosão de acordo com os critérios de Denis e a classificação de Magerl, subtipo $\mathrm{A}^{(24)}$, com tempo de lesão menor do que 10 dias à admissão, de uma 
única vértebra, sem alteração neurológica, com pelo menos seis meses de acompanhamento com tratamento conservador, submetidos à confecção de gesso antigravitacional ou órtese toracolombossacra (TLSO) e que responderam à convocação para reavaliação clínica, por meio de questionários e radiografias.

Foram excluídos os casos de fraturas em duas ou mais vértebras, sem documentação completa (prontuário, radiografia e TC), que não responderam à convocação e com fratura patológica, vítimas de ferimento por arma de fogo ou com déficit neurológico. Também foram excluídos os pacientes que foram admitidos após mais de 10 dias da data da fratura (fraturas tardias).

Os pacientes foram avaliados com base no questionário de qualidade de vida Short-Form 36 (SF-36) ${ }^{(25)}$, sendo utilizada a sua validação em português. Também foram aplicadas as escalas de dor e trabalho de Denis ${ }^{(1)}$ (Tabelas 1 e 2) e avaliado o quadro clínico neurológico conforme a classificação de Frankel ${ }^{(26)}$ (Tabela 3).

Tabela 1 - Escala funcional de dor segundo Denis ${ }^{(1)}$

\begin{tabular}{c|l}
\hline Pontuação & \multicolumn{1}{c}{ Critério de escala de dor } \\
\hline 1 & Sem dor \\
\hline 2 & Dor mínima, sem uso de medicação \\
\hline 3 & Dor moderada, com uso ocasional de medicação \\
\hline 4 & Dor moderada a grave, com uso constante de medicação \\
\hline 5 & Dor grave, com uso crônico de medicação \\
\hline
\end{tabular}

Fonte: Traduzido de Denis F. ${ }^{(1)}$

Tabela 2 - Escala funcional de trabalho segundo Denis ${ }^{(1)}$

\begin{tabular}{c|l}
\hline Potuação & \multicolumn{1}{c}{ Critério de escala de trabalho } \\
\hline 1 & Retorno ao trabalho pesado \\
\hline 2 & Retorno ao trabalho sedentário, sem restrição de peso \\
\hline 3 & Retorno ao trabalho, mas mudou de atividade \\
\hline 4 & Retorno ao trabalho, porém por meio período \\
\hline 5 & Incapaz para o trabalho \\
\hline
\end{tabular}

Fonte: Traduzido de Denis F.(1)

Tabela 3 - Classificação de Frankel ${ }^{(26)}$

\begin{tabular}{c|c|c}
\hline Classificação & Motricidade & Sensibilidade \\
\hline A & ausente & Ausente \\
\hline B & ausente & Presente \\
\hline C & presente não útil & Presente \\
\hline D & presente útil & Presente \\
\hline E & Normal & Normal \\
\hline
\end{tabular}

Fonte: Traduzido de: Frankel HL et al. ${ }^{(26)}$
A mensuração da cifose foi realizada segundo o método de $\operatorname{Cobb}^{(27)}$ no momento da admissão no hospital e no final do acompanhamento. O percentual de acometimento do canal vertebral foi medido na TC com régua milimétrica, segundo método descrito por Trafton e Boyd ${ }^{(28)}$, utilizando como valor normal a média das vértebras adjacentes.

Para as análises estatísticas deste estudo, foi considerado nível de significância de 5\%. Foi utilizado o programa SPSS (Statistical Package for Social Sciences), em sua versão 13.0, para a obtenção dos resultados.

Foi aplicada análise de correlação de Spearman, com o intuito de verificar o grau de relacionamento entre as variáveis de interesse, quando estudadas par a par. Foi realizada também uma avaliação adicional no subgrupo de pacientes com deformidade grave (cifose final $\geq 30^{\circ}$ ), em que foram comparadas as médias do SF-36, Denis dor e Denis trabalho desses com o restante da população do estudo, por meio do teste de Mann-Whitney. Utilizou-se o teste de Wilcoxon para averiguar a diferença entre a cifose inicial e do final do acompanhamento (Tabela 4).

Tabela 4 - Correlação entre cifose final e escore dor, SF-36 e Escala de Dor e Trabalho de Denis ${ }^{(1)}$

\begin{tabular}{c|c|c}
\hline Variável / cifose final & $\begin{array}{c}\text { Coeficiente } \\
\text { de correlação }\end{array}$ & $\mathbf{P}$ \\
\hline Escore_dor & 0,563 & $<0,001^{*}$ \\
\hline SF-36 & $-0,120$ & 0,484 \\
\hline Denis dor & 0,149 & 0,386 \\
\hline Denis trabalho & 0,281 & 0,097 \\
\hline
\end{tabular}

Preencheram o critério de inclusão para esta amostra 36 pacientes. A média de idade dos pacientes estudados foi de 50,83 anos, com mínimo de 13 e máximo de 83 anos, sendo 20 do sexo masculino e 16 do feminino. O mecanismo de trauma foi a queda de altura em 24 pacientes, acidente automobilístico em cinco, queda da própria altura em cinco casos e soterramento em um caso. Quanto à vértebra fraturada, observaram-se: T11 em um caso, T12 em sete casos, L1 em 15 pacientes, L2 em 11 pacientes e dois em L3. Todos os pacientes sem lesão neurológica na internação hospitalar (Frankel E). O tempo de seguimento médio foi de 66,38 meses, variando de 13 a 185 meses. O comprometimento do canal vertebral foi em média de 19,25\%, com variação de 5 a $60 \%$. 


\section{RESULTADOS}

Na avaliação radiográfica, a média de cifose inicial foi de $12,16^{\circ}$, com variação de 0 a $40^{\circ}$ e a média de cifose final foi de $13,41^{\circ}$, variando de 0 a $45^{\circ}$. Não houve diferença significante entre os valores de Cobb iniciais e do final do acompanhamento. (12,1 vs 13,4; $\mathrm{p}>0,05)$. A diferença desses valores variou de -11 a $45^{\circ}$, com média de $1,38^{\circ}$ (Tabela 5). Todos os pacientes apresentaram-se, no último exame, sem déficit neurológico (Frankel E) (Figura 1).

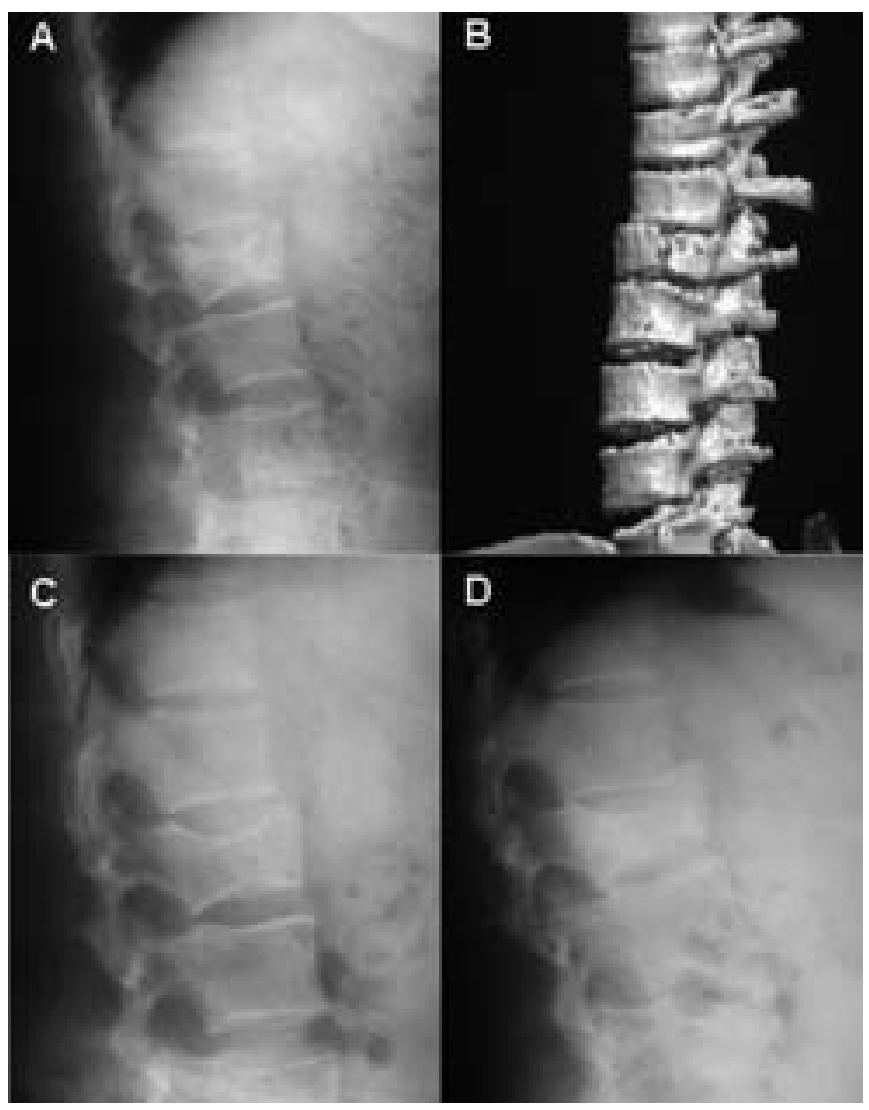

Figura 1 - A) Portadora de fratura explosão da primeira vértebra lombar. B) Observar a fragmentação do corpo vertebral na TC com recontrução. C) Melhora do alinhamento toracolombar após a confecção do gesso em hiperextensão. D) Apesar da perda da correção, a paciente encontra-se assintomática

Tabela 5 - Correlação entre cifose final e subdivisões do SF-36(25)

\begin{tabular}{c|c|c}
\hline Variávell cifose final & Coeficiente de correlação & $\mathbf{P}$ \\
\hline Capacidade funcional & $-0,195$ & 0,253 \\
\hline Aspectos físicos & $-0,180$ & 0,294 \\
\hline Dor & 0,126 & 0,463 \\
\hline Estado geral saúde & 0,014 & 0,936 \\
\hline Vitalidade & 0,060 & 0,728 \\
\hline Aspectos sociais & $-0,055$ & 0,751 \\
\hline Aspectos emocionais & 0,140 & 0,415 \\
\hline Saúde mental & 0,032 & 0,852 \\
\hline
\end{tabular}

Em relação à dor, cinco pacientes estavam sem dor, 15, com dor leve, 13, com dor moderada, e três, com dor grave. Quanto ao trabalho, 12 pacientes retornaram ao trabalho pesado, 10 voltaram ao trabalho sedentário, oito mudaram de atividade, um retornou por meio período e cinco encontravam-se incapazes para o trabalho.

Observou-se correlação positiva ( $\mathrm{p}<0,05 ; \mathrm{r}=0,563$ ) entre a cifose final e escore de dor (VAS); entretanto, essa correlação foi fraca (Figura 2). Não se verificou correlação entre cifose final e a pontuação dos questionários SF36 e Denis, incluindo suas subdivisões (Tabela 6).

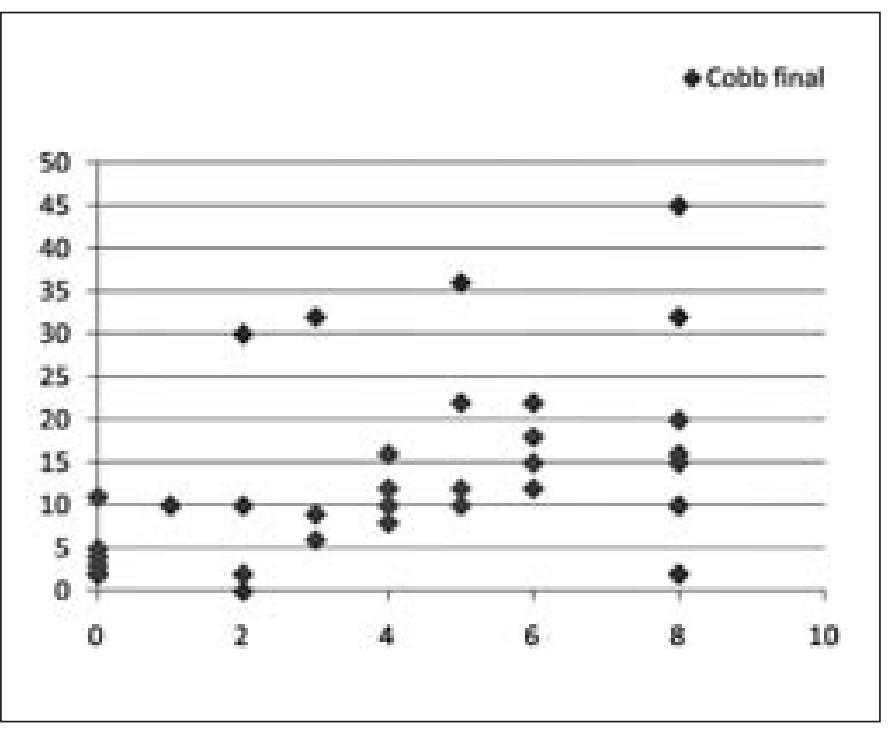

Figura 2 - Correlação entre o VAS e a cifose final

Tabela 6 - Correlação entre cifose final e subdivisões do SF-36(25)

\begin{tabular}{c|c|c}
\hline Variável/ cifose final & Coeficiente de correlação & $\mathbf{P}$ \\
\hline Capacidade funcional & $-0,195$ & 0,253 \\
\hline Aspectos físicos & $-0,180$ & 0,294 \\
\hline Dor & 0,126 & 0,463 \\
\hline Estado geral saúde & 0,014 & 0,936 \\
\hline Vitalidade & 0,060 & 0,728 \\
\hline Aspectos sociais & $-0,055$ & 0,751 \\
\hline Aspectos emocionais & 0,140 & 0,415 \\
\hline Saúde mental & 0,032 & 0,852 \\
\hline
\end{tabular}

Não houve diferença entre as médias das pontuações dos questionários SF-36 ( $\mathrm{p}=0,450)$ e Denis dor ( $\mathrm{p}=0,142)$ e Denis trabalho $(\mathrm{p}=0,081)$ nos pacientes com cifose maior ou igual a $30^{\circ}$ (cinco pacientes) ou menor do que $30^{\circ}$ (31 pacientes). 


\section{DISCUSSÃO}

O tratamento da fratura toracolombar tipo explosão é um tema controverso. Um dos argumentos a favor do tratamento conservador é a tese do menor risco de o paciente desenvolver cifose pós-traumática dolorosa $^{(1,5-7,29)}$. Verificamos que a avaliação do tratamento conservador em geral é realizada com base em critérios radiográficos. Além disso, a maioria dos trabalhos não avalia desfechos funcionais com escalas validadas do tipo transcultural. Algumas séries de casos não sugerem correlação entre a cifose pós-traumática e sintomatologia após o tratamento conservador dessas fraturas; contudo, muitos trabalhos apresentam número pequeno de pacientes, critérios subjetivos de avaliação ou não consideram os subtipos de Magerl ${ }^{(24)}$ nesses pacientes (Quadros 1 e 2) $)^{(8,14,16,23)}$ Ademais, há sugestão de alguns autores de que a cifose acima de $30^{\circ}$ seria sinal de piores desfechos funcionais e, por isso, seria

Quadro 1 - Distribuição dos pacientes portadores de fratura toracolombar explosão internados entre 1991 e 2008 na SCMSP

\begin{tabular}{|c|c|c|c|c|c|c|c|c|c|c|c|c|}
\hline Paciente & Sexo & Idade & Trauma & Nível & Meses & $\begin{array}{l}\text { Cifose } \\
\text { inicial }\end{array}$ & $\begin{array}{l}\text { Cifose } \\
\text { final }\end{array}$ & $\begin{array}{c}\text { Progressão da } \\
\text { deformidade }\end{array}$ & VAS & SF-36 & Denis dor & $\begin{array}{l}\text { Denis } \\
\text { trabalho }\end{array}$ \\
\hline 1 & $\mathrm{~F}$ & 63 & Queda de altura & L2 & 121 & 13 & 2 & -9 & 8 & 86 & 5 & 3 \\
\hline 2 & $\mathrm{~F}$ & 68 & Queda de altura & L1 & 70 & 7 & 10 & 3 & 8 & 98 & 4 & 2 \\
\hline 3 & M & 30 & Ac. automóvel & T12 & 46 & 10 & 9 & -1 & 3 & 98 & 2 & 1 \\
\hline 4 & M & 56 & Queda de altura & L3 & 35 & 3 & 6 & 3 & 3 & 98 & 2 & 1 \\
\hline 5 & M & 63 & Queda de altura & L2 & 52 & 0 & 2 & 2 & 0 & 89 & 2 & 1 \\
\hline 6 & M & 75 & Ac. automóvel & L2 & 60 & 3 & 0 & -3 & 2 & 79 & 2 & 2 \\
\hline 7 & $\mathrm{~F}$ & 65 & Queda de altura & L1 & 120 & 9 & 18 & 9 & 6 & 83 & 4 & 3 \\
\hline 8 & $\mathrm{~F}$ & 38 & Queda de altura & T12 & 114 & 12 & 15 & 3 & 6 & 84 & 1 & 2 \\
\hline 9 & $\mathrm{~F}$ & 53 & Queda de altura & L2 & 106 & 14 & 10 & -4 & 5 & 85 & 3 & 2 \\
\hline 10 & M & 35 & Queda de altura & T12 & 42 & 10 & 3 & -7 & 0 & 81 & 1 & 1 \\
\hline 11 & $\mathrm{~F}$ & 59 & Queda de altura & T12 & 185 & 22 & 11 & -11 & 0 & 98 & 2 & 3 \\
\hline 12 & $\mathrm{~F}$ & 50 & Ac. automóvel & L1 & 128 & 1 & 4 & 3 & 0 & 99 & 5 & 2 \\
\hline 13 & $\mathrm{~F}$ & 57 & Esmagamento & L1 & 66 & 10 & 15 & 5 & 8 & 99 & 1 & 3 \\
\hline 14 & M & 41 & Queda de altura & L1 & 51 & 20 & 22 & 2 & 6 & 82 & 3 & 1 \\
\hline 15 & $\mathrm{~F}$ & 28 & Queda de altura & L2 & 45 & 10 & 2 & -8 & 0 & 100 & 4 & 4 \\
\hline 16 & M & 83 & Queda de altura & L1 & 39 & 3 & 5 & 2 & 0 & 100 & 4 & 5 \\
\hline 17 & $\mathrm{~F}$ & 49 & Queda de altura & L2 & 47 & 40 & 32 & -8 & 3 & 100 & 3 & 2 \\
\hline 18 & M & 13 & Ac. automóvel & L1 & 14 & 3 & 2 & -1 & 0 & 100 & 1 & 1 \\
\hline 19 & M & 31 & Queda de altura & L2 & 13 & 0 & 2 & 2 & 2 & 100 & 2 & 2 \\
\hline 20 & M & 60 & Queda de altura & L1 & 50 & 10 & 16 & 6 & 4 & 90 & 2 & 5 \\
\hline 21 & $\mathrm{~F}$ & 74 & Queda de altura & L2 & 28 & 4 & 20 & 16 & 8 & 87 & 3 & 3 \\
\hline 22 & $\mathrm{~F}$ & 56 & Queda de altura & T12 & 100 & 32 & 45 & 13 & 8 & 93 & 4 & 3 \\
\hline 23 & M & 51 & Queda de altura & T12 & 30 & 8 & 8 & 0 & 4 & 84 & 2 & 2 \\
\hline 24 & M & 51 & Queda de altura & L1 & 70 & 10 & 10 & 0 & 4 & 98 & 2 & 2 \\
\hline 25 & M & 44 & Queda de altura & L1 & 66 & 26 & 32 & 6 & 8 & 68 & 4 & 3 \\
\hline 26 & M & 50 & Queda de altura & L3 & 48 & 4 & 10 & 6 & 1 & 95 & 2 & 1 \\
\hline 27 & $\mathrm{~F}$ & 48 & Queda ao solo & T12 & 31 & 16 & 22 & 6 & 5 & 89 & 3 & 2 \\
\hline 28 & M & 70 & Queda ao solo & L1 & 96 & 10 & 16 & 6 & 8 & 84 & 2 & 5 \\
\hline 29 & M & 36 & Queda de altura & L1 & 96 & 36 & 30 & -6 & 2 & 100 & 2 & 5 \\
\hline 30 & M & 35 & Queda de altura & L1 & 36 & 6 & 12 & 6 & 4 & 90 & 2 & 1 \\
\hline 31 & $\mathrm{~F}$ & 73 & Queda ao solo & L1 & 36 & 12 & 12 & 0 & 6 & 90 & 1 & 1 \\
\hline 32 & $\mathrm{~F}$ & 70 & Queda ao solo & L1 & 62 & 8 & 12 & 4 & 5 & 100 & 2 & 1 \\
\hline 33 & M & 51 & Queda de altura & L2 & 93 & 10 & 10 & 0 & 8 & 89 & 3 & 5 \\
\hline 34 & $\mathrm{~F}$ & 29 & Queda de altura & T11 & 25 & 30 & 36 & 6 & 5 & 96 & 3 & 3 \\
\hline 35 & $M$ & 40 & Queda de altura & L2 & 60 & 10 & 10 & 0 & 2 & 96 & 2 & 1 \\
\hline 36 & $M$ & 35 & Ac. automóvel & L2 & 109 & 16 & 12 & -4 & 4 & 90 & 3 & 1 \\
\hline
\end{tabular}


FRATURA TORACOLOMBAR DO TIPO EXPLOSÃO: CORRELAÇÃO ENTRE CIFOSE

Quadro 2 - Série de casos de portadores de fratura toracolombar explosão

\begin{tabular}{|c|c|c|c|c|c|}
\hline Autores & Cobb inicial & Cobb final & $\mathrm{n}$ & Questionário utilizado & Tipo de estudo \\
\hline Weinstein $^{16}$ & $26,4^{\circ}$ & $16,8^{\circ}$ & 42 & VAS, escala de dor, questionário funcional próprio & Retrospectivo \\
\hline Avanzi $^{8}$ & $11,1^{\circ}$ & $12,7^{\circ}$ & 17 & Escala de dor, Denis & Retrospectivo \\
\hline Cantor $^{14}$ & $19^{\circ}$ & $20^{\circ}$ & 33 & Denis, questionário funcional próprio & Prospectivo \\
\hline Mumford ${ }^{23}$ & $16,24^{\circ}$ & $20,12^{\circ}$ & 41 & $\begin{array}{c}\text { VAS, Roland \& Morris, função - questionário de } \\
\text { atividades da vida diária }\end{array}$ & prospectivo \\
\hline Chow ${ }^{22}$ & $5,3^{\circ}$ & $7,6^{\circ}$ & 24 & Denis modificado (dor), questionário funcional próprio & Retrospectivo \\
\hline Shen $^{21}$ & $20^{\circ}$ & $24^{\circ}$ & 38 & Denis & Retrospectivo \\
\hline Gertzbein ${ }^{27}$ & $12,4^{\circ}$ & $13,9^{\circ}$ & 96 & $\begin{array}{l}\text { Função - Frankel, Motor Score Index, (dor) questionário } \\
\text { baseado em uso de medicação }\end{array}$ & Prospectivo \\
\hline
\end{tabular}

um dos indicativos do tratamento operatório ${ }^{(9-17,29)}$, mas observamos escassez de avaliação em separado nesse grupo de pacientes submetidos ao tratamento conservador. Gertzbein ${ }^{(29)}$, com série multicêntrica de 641 pacientes, associou cifose com dor; entretanto, o grupo de avaliação foi heterogêneo (545 pacientes operados) e não foram avaliados em separado os portadores de cifose acima de $30^{\circ}$.

Ao contrário dos achados de Weinstein et al ${ }^{(16)}$ e Mumford et $a l^{(23)}$, observamos correlação entre o EVA e a cifose final. Mas, vale ressaltar que essa correlação foi fraca. Esse instrumento de avaliação, que vai de 0 a 10, na comparação com as escalas de Denis, que variam de 1 a 5 , pode facilitar a análise numérica de estimativa estatística da dor nesses pacientes. Estudos futuros com série maior de casos podem mostrar tendência não observada nos estudos atuais com menor número de pacientes.

Para avaliação da qualidade de vida e dor dos pacientes do nosso trabalho foram utilizados os questionários Short-Form 36 e a escala EVA. Esses instrumentos são validados para uso em nosso meio e de grande valor para análise do resultado do tratamento centrado no paciente, e não apenas com base em critérios radiográficos. De forma similar aos nossos achados, houve falta de correlação evidente entre a cifose e sintomatologia ou função na coluna após o tratamento conservador nas séries de casos levantadas ${ }^{(16,22,23)}$.

Para comparar com nossos achados de $86,1 \%$ de retorno às atividades laborativas, Mumford et $\mathrm{al}^{(23)}$, em seu estudo prospectivo de 41 pacientes tratados de modo conservador, acompanhados por dois anos em média, verificaram que 90,3\% deles retornaram ao seu trabalho. Outros trabalhos ${ }^{(8,14,16,21,22)}$ mostraram valores de 75 a $95 \%$.
O padrão de resultados nesta série é consistente com aqueles encontrados na literatura em relação à dor. Dos 33 pacientes estudados por Cantor et $a l^{(14)}, 18$ (65\%) apresentavam algum grau de dor decorrente da fratura, sendo que nesta série $61 \%$ dos pacientes referiam dor residual.

Discute-se a respeito da progressão média da deformidade, porém, inexiste análise estatística mais detalhada desse achado. Verificamos média de $1,38^{\circ}$ de progressão da deformidade, mas sem significância estatística dos achados. Isso sugere que a cifose no final do acompanhamento é similar à encontrada na radiografia inicial. Outra vantagem deste estudo é o foco nas fraturas subtipo A3. A maioria dos autores apenas considera a classificação de Denis com base na imagem da TC, que na avaliação mais detalhada pode apresentar componentes dos subtipos A, B ou C de Magerl ${ }^{(24)}$, com características biomecânicas distintas.

Outra questão que deve ser discutida é a avaliação em separado do grupo de portadores de cifose acima de $30^{\circ}$, um dos critérios de cirurgia para alguns autores pela possibilidade do desenvolvimento de cifose pós-traumática dolorosa. Apesar dessa opinião, não há avaliação objetiva desse grupo de pacientes tratados de modo conservador nos trabalhos levantados ${ }^{(1,5-7,30)}$. Não houve piores resultados funcionais nesses pacientes, porém, devemos enfatizar que o número pequeno de casos (cinco) pode dificultar a análise estatística definitiva. Estudos com foco especial nesses pacientes devem ser realizados.

As limitações observadas neste estudo foram seu caráter retrospectivo, com inerente dificuldade de acompanhamento das variáveis que podem confundir os achados - aderência ao uso de órteses, compensação financeira e outras comorbidades - e a falta de grupo 
controle de comparação de pacientes operados. Outros desenhos de estudos devem ser realizados para melhorar o nível de evidência desses resultados. Contudo, diante da falta desses estudos, justifica-se a importância de pesquisa tipo série de casos.

\section{CONCLUSÃO}

Não foi encontrada evidente correlação entre a cifose pós-traumática e a progressão da deformidade com a dor do paciente, após tratamento conservador de portadores de fratura toracolombar tipo explosão e subtipo DenisA3 sem lesão neurológica.

\section{AGRADECIMENTO}

Agradecemos ao Núcleo de Apoio à Publicação da Faculdade de Ciências Médicas da Santa Casa de São Paulo (NAP-SC) o suporte técnico-científico à publicação deste manuscrito.

\section{REFERÊNCIAS}

1. Denis F. The three column spine and its significance in the classification of acute thoracolumbar spinal injuries. Spine. 1983;8(8):817-31.

2. Kraemer WJ, Schemitsch EH, Lever J, McBroom RJ, McKee MD, Waddell JP. Functional outcome of thoracolumbar burst fractures without neurological deficit. J Orthop Trauma. 1996;10(8):541-4.

3. Thomas KC, Bailey CS, Dvorak MF, Kwon B, Fisher C. Comparison of operative and nonoperative treatment for thoracolumbar burst fractures in patients without neurological deficit: a systematic review. J Neurosurg Spine. 2006;4(5):351-8.

4. Knight RQ, Stornelli DP, Chan DP, Devanny JR, Jackson KV. Comparison of operative versus nonoperative treatment of lumbar burst fractures. Clin Orthop Relat Res. 1993;(293):112-21.

5. Watson Jones R. Manipulative reduction of crush fractures of the spine. Br Med J. 1931;1(3659):300-2.

6. Nicoll EA. Fractures of the dorso-lumbar spine. J Bone Joint Surg Am. 1949;31B(3):376-94.

7. Holdsworth F. Fractures, dislocations and fracture-dislocations of the spine. J Bone Joint Surg Am. 1970;52(8):1534-51.

8. Avanzi O, Chih LY, Meves R, Caffaro MFS, Bueno RS, Freitas MMF. Fratura toracolombar tipo explosão: resultados do tratamento conservador. Rev Bras Ortop. 2006;41(4):109-15.

9. Tezer M, Erturer RE, Ozturk C, Ozturk I, Kuzgun U. Conservative treatment of fractures of the thoracolumbar spine. Int Orthop. 2005;29(2):78-82.

10. Tropiano P, Huang RC, Louis CA, Poitout DG, Louis RP. Functional and radiographic outcome of thoracolumbar and lumbar burst fractures managed by closed orthopaedic reduction and casting. Spine. 2003;28(21):2459-65.

11. Shen WJ, Liu TJ, Shen YS. Nonoperative treatment verus posterior fixation for thoracolumbar junction burst fractures without neurologic deficit. Spine. 2001;26(9):1038-45.

12. Hitchon PW, Torner JC, Haddad SF, Follett KA. Management options in thoracolumbar burst fractures. Surg Neurol. 1998;49(6):619-27.

13. Domenicucci M, Preite R, Ramieri A, Ciappetta P, Delfini R, Romanini L. Thoracolumbar fractures without neurosurgical involvement: surgical or conservative treatment? J Neurosurg Sci. 1996;40(1):1-10.

14. Cantor JB, Lebwohl NH, Garvey T, Eismont FJ. Nonoperative management of stable thoracolumbar burst fractures with early ambulation and bracing. Spine. 1993;18(8):971-6

15. Reid DC, Hu R, Davis LA, Saboe LA. The nonoperative treatment of burst fractures of the thoracolumbar junction. J Trauma. 1988;28(8):1188-94.
16. Weinstein JN, Collalto P, Lehmann TR. Thoracolumbar burst fractures treated conservatively: a long-term follow-up. Spine. 1988;13(1):33-8.

17. McEvoy RD, Bradfort DS. The management of burst fractures of the thoracic and lumbar spine. Experience in 53 patients. Spine. 1985;10(7):631-7.

18. Moller A, Hasserius R, Redlund-Johnell I, Ohlin A, Karlsson MK. Nonoperative treated burst fractures of the thoracic and lumbar spine in adults: a 23 to 41 year follow-up. Spine J. 2007;7(6):701-7.

19. Agus H, Kayali C, Arslantas M. Nonoperative treatment of burst-type thoracolumbar vertebra fractures: clinical and radiological results of 29 patients. Eur Spine J. 2005;14(6):536-40.

20. Wood K, Buttermann G, Mehbod A, Garvey T, Jhanjee R, Sechriest V. Operative compared with nonoperative treatment of a thoracolumbar burst fracture without neurological deficit. A prospective, randomized study. J Bone Joint Surg Am. 2003;85A(5):773-81.

21. Shen WJ, Shen YS. Nonsurgical treatment of three-column thoracolumbar junction burst fractures without neurologic deficit. Spine. 1999;24(4):412-5.

22. Chow GH, Nelson BJ, Gebhard JS, Brogman JL, Brown CW, Donaldson DH. Functional outcome of thoracolumbar burst fractures managed with hyperextension casting or bracing and early mobilization. Spine. 1996;21(18):2170-5.

23. Mumford J, Weinstein JN, Spratt KF, Goel VK. Thoracolumbar burst fractures. The clinical efficacy and outcome of nonoperative managment. Spine. 1993;18(8):955-70.

24. Magerl F, Aebi M, Gertzbein SD, Harms J, Nazarian S. A comprehensive classification of thoracic and lumbar injuries. Eur Spine J. 1994;3(4):184-201.

25. Ware JE Jr, Sherbourne CD. The MOS 36-item short-form health survey (SF-36). I. Conceptual framework and item selection. Med Care. 1992;30(6):473-83.

26. Frankel HL, Hancock DO, Hyslop G, Melzak J, Michaelis LS, Ungar GH, et al. The value of postural reduction in the initial management of closed injuries of the spine with paraplegia and tetraplegia. I. Paraplegia. 1969;7(3):179-92.

27. Harrison DE, Cailliet R, Harrison DD, Janik TJ, Holland B. Reliability of centroid, Cobb, and Harrison posterior tangent methods: which to choose for analysis of thoracic kyphosis. Spine. 2001;26(11):E227-34.

28. Trafton PG, Boyd CA Jr. Computed tomography of thoracic and lumbar spine injuries. J Trauma. 1984;24(6):506-15.

29. Gertzbein SD. Scoliosis Research Society: multicenter spine fracture study. Spine 1992;17(5):528-40.

30. Million R, Hall W, Nilsen KH, Baker RD, Jayson MIV. Assessment of the progress of the back pain patient. Spine. 1982;7:204-12. 